\title{
Exposición periconcepcional a factores ambientales y de consumo en padres y cuidadores de niños menores de 5 años en Santander
}

\author{
Periconceptional Exposure to Environmental and Consumer \\ Factors in Parents and Caregivers of Children Under 5 Years Old \\ in Santander
}

\section{Exposição periconcepcional aos fatores ambientais e do consumo dos pais e dos cuidadores de crianças menores de 5 anos de idade no estado de Santander}

John Freddy Arguello-Duarte, Enf., MSc. *

Diana Carolina Jaimes, MD., MSc. **

Jhyld Carolaind Camacho-Barbosa, ND., MSc. ***

Pilar Abreu-Peralta, Enf., MSc. ****

\section{Resumen}

Introducción: El desarrollo de estrategias que faciliten el cribado, diagnóstico y abordaje de factores medioambientales durante el embarazo y la lactancia, etapas donde la salud materno-infantil está expuesta de manera activa o pasiva a diferentes agentes; requiere implementar formatos facilitadores de abordaje preventivo. Objetivo: Identificar los factores medioambientales, desde la etapa periconcepcional hasta la infancia, utilizando como herramienta compartida la hoja verde de salud ambiental. Metodología: Estudio

\author{
Martha Isabel Robles-Carreño, Enf., Esp. ***** \\ Luisa Fernanda Suarez-Mejía, Est. ****** \\ Karol Yadira Mantilla-Carreño, Est. $* * * * * *$ \\ Lina Zuleima Jaimes-Gómez, Est. ******
}

observacional, descriptivo de corte transversal. La muestra fue de 204 mujeres gestantes y/o lactantes entre 13-46 años de edad, de municipios de Santander. Se realizó un análisis estadístico descriptivo, de acuerdo a la naturaleza de las variables. Resultados: La edad promedio fue de 25.12 años $(\mathrm{DE}=5.94)$. El promedio de semanas gestacionales fue de 36.65 (DE=5.63). Consumo de alcohol preconcepciónal del $48.52 \%(n=99)$, donde $91.91 \%(n=91)$ consumió cerveza con frecuencia de consumo pregestacional de 4.96 cervezas/mes $(D E=4.58)(n=64)$. El consumo de cigarro materno en etapas periconcepcionales, fue de $4.90 \%$ con promedio de 2.03

* Enfermero, magíster en Neurociencias y Biología del Comportamiento, docente Universidad Autónoma de Bucaramanga, Santander, Colombia.

** Médica, magíster en Epidemiología, coordinadora general red iberoamericana de salud ambiental infantil (SAMBI), Colombia.

*** Nutricionista y Dietista, magíster en Epidemiología, investigadora red iberoamericana de salud ambiental infantil (SAMBI), Colombia

**** Enfermera, magíster en Educación, docente Universidad Autónoma de Bucaramanga, Santander, Colombia.

***** Enfermera, especialista en Cuidado Crítico Neonatal, docente Universidad Autónoma de Bucaramanga, Santander, Colombia

******Estudiante de Enfermería, Universidad Autónoma de Bucaramanga, Santander, Colombia.

Correspondencia: John Freddy Arguello Duarte. Calle 157 No. 19-55, Cañaveral Parque, Universidad Autónoma de Bucaramanga Campus El Bosque, dirección programa de Enfermería. Celular: +57 3008047115. E-mail: jarguello451@unab.edu.co. 
cigarros/día (DE=4.35). Conclusiones: Este estudio aporta datos del ámbito local, y traslada una herramienta válida de promoción y prevención en salud medioambiental, por lo que la aplicación de este instrumento permitiría el abordaje integral de la consulta de atención madre-hijo. [ArguelloDuarte JF, Jaimes DC, Camacho-Barbosa JC, Abreu-Peralta $P$, Robles-Carreño MI, Suarez-Mejía LF, Mantilla-Carreño KY, Jaimes-Gómez LZ. Exposición periconcepcional a factores ambientales y de consumo en padres y cuidadores de niños menores de 5 años en Santander. MedUNAB 2017; 20(2): 131-138].

Palabras claves: Salud Ambiental; Embarazo; Lactancia; Crianza del niño; Alcoholismo; Consumo de Bebidas Alcohólicas.

\section{Abstract}

Introduction: The development of strategies that facilitate the screening, diagnosis and approach of environmental factors during pregnancy and lactation, stages in which maternal and child health is exposed in an active or passive way to different agents; it requires implementing facilitative formats of a preventive approach. Objective: To identify environmental factors, from the periconceptional stage to childhood by using the "Green Page" of environmental health as a shared tool. Methodology: This is an observational and descriptive cross-sectional study. The sample consisted of 204 pregnant women and / or infants between 13 - 46 years old, from different municipalities in Santander. According to the nature of the variables, a descriptive statistical analysis was carried out. Results: The average age was 25.12 years $(S D=5.94)$. The pregnancy weeks average was $36.65(S D=$ 5.63). Preconception of alcohol intake of $48.52 \%(n=99)$, in which $91.91 \%(n=91)$ consumed beer with a frequency of pre-pregnancy intake of 4.96 beers / month $(S D=4.58)(n=$ 64). The cigar intake of women in periconceptional stages was $4.90 \%$ with an average of 2.03 cigars / day $(S D=4.35)$. Conclusions: This study provides data from the local area, and moves a valid tool promotion and prevention tool to environmental health, so the application of this instrument would allow the integral approach of the mother-child care consultation. [Arguello-Duarte JF, Jaimes DC, CamachoBarbosa JC, Abreu-Peralta P, Robles-Carreño MI, Suarez-

\section{Introducción}

La contaminación de los ecosistemas y la creciente preocupación social de los efectos potencialmente lesivos en la salud, hizo que la Organización Mundial de la Salud (OMS), definiera la salud medioambiental como: a) aspectos que incluyen la calidad de vida, dadas las interacciones de agentes medioambientales de tipo químicos, físicos, psíquicos, sociales y biológicos; y b) aspectos teórico-prácticos que controlan, modifican, evalúan, corrigen y previenen los factores o agentes medioambientales que, potencialmente, afecten de manera negativa la salud de todas las generaciones (1).

Por lo tanto, para mejorar el estado de salud general se requiere un abordaje integral y holístico que gestione los
Mejía LF, Mantilla-Carreño KY, Jaimes-Gómez LZ. Periconceptional Exposure to Environmental and Consumer Factors in Parents and Caregivers of Children Under 5 Years Old in Santander. MedUNAB 2017; 20(2): 131-138].

Key Words: Environmental Health; Pregnancy; Lactation; Child Rearing; Alcoholism; Alcohol Drinking.

\section{Resumo}

Introdução: O desenvolvimento de estratégias que facilitem a triagem, o diagnóstico e a abordagem dos fatores ambientais durante a gestação e a lactação, etapas em que a saúde materno-infantil é exposta de maneira ativa ou passiva a diferentes agentes; requer a implementação de formatos facilitadores d'abordagem preventiva. Objetivo: Identificar fatores ambientais, desde a fase periconcepcional até a infância, utilizando a folha verde da saúde ambiental como ferramenta compartilhada. Metodologia: De tipo descritivo, de observação, e transversal. A amostra realizada foi de 204 gestantes e/ ou lactantes entre 13 e 46 anos de idade, de diferentes municípios de Santander. De acordo com a natureza das variáveis, se realizou uma análise estatística descritiva. Resultados: A idade média foi de 25,12 anos (DP $=5,94)$. O pro médium de semanas, nas gestantes foi de 36,65 (DP = 5,63). O consumo de álcool na pré concepção é de $48,52 \%$ ( $n=99)$, entre ellas, 91,91\% ( $n=91)$ beberam cerveja com frequência de 4,96 cervejas ao mês ( $D E=4,58)$ $(n=64)$. As mães fumadoras no periodo da pré concepção foi de 4,90\%, com um pro médium de 2,03 cigarros por dia (DP = $4,35)$. Conclusões: O estudo apresenta os dados da região e usa uma ferramenta válida de promoção e prevenção em saúde ambiental, para que a aplicação deste instrumento permita a abordagem integral da consulta e dos cuidados materno-infantil, nos casos atendidos. [Arguello-Duarte JF, Jaimes DC, Camacho-Barbosa JC, Abreu-Peralta P, RoblesCarreño MI, Suarez-Mejía LF, Mantilla-Carreño KY, JaimesGómez LZ. Exposição periconcepcional aos fatores ambientais e do consumo dos pais e dos cuidadores de crianças menores de 5 anos de idade no estado de Santander. MedUNAB 2017; 20(2): 131-138].

Palavras-chave: Saúde Ambiental; Gravidez; Lactação; Educação Infantil; Alcoolismo; Consumo de Bebidas Alcoólicas.

problemas de salud relacionados al ambiente, educando las familias en prevención de la exposición (2) y desarrollando estrategias que faciliten el cribado, diagnóstico y abordaje de la salud, durante todas las etapas críticas del individuo en desarrollo: desde la etapa preconcepcional hasta el final de la adolescencia (3-7).

El embarazo, la lactancia y el periodo periconcepcional (abarca los tres meses previos a la fecundación) son etapas vulnerables a agentes medioambientales, en las cuales, la salud materno-infantil puede afectarse por la exposición activa o pasiva a diferentes factores (8-13). Durante estas etapas, las sustancias psicoactivas legales e ilegales constituyen una de las exposiciones a tóxicos medioambientales, con efectos severos en el desarrollo infantojuvenil potencialmente prevenibles en el 100\% de los casos 
$(10,14)$, por lo cual, en una perspectiva medioambiental global, las drogas tiene un rol relevante en la salud del binomio madre-hijo. Por otra parte, el alcohol es el teratógeno más frecuente en la población general, por lo que su temprana detección, disminuye el riesgo fetal y de morbilidad infantil. Las sustancias psicoactivas en general, junto con los pesticidas, son los factores de riesgo más frecuentes y relevantes durante el embarazo y la lactancia, y se constituyen exposiciones derivadas del trabajo, hobbies o ambientales (agua, aire y calidad de alimentos) $(10,14,15)$.

Para la detección y manejo de estas exposiciones ambientales, la OMS ha desarrollado una herramienta conocida como "La hoja verde" (16). Esta herramienta ha sido reproducida y ajustada en diferentes contextos culturales, para la evaluación del riesgo medioambiental pediátrico (17-19); también se ha adaptado a la salud reproductiva para su uso durante el periodo pre-gestacional, gestacional y de lactancia. Está compuesta de preguntas básicas y concisas que permiten detectar y, posteriormente, abordar los factores de riesgo medioambientales durante la etapa periconcepcional, embarazo, lactancia y periodo de crianza teniendo en cuenta todos los escenarios potenciales de exposición de la pareja y sus fuentes (actividades ocupacionales, tratamientos médicos y hábitos como el consumo de sustancias psicoactivas, que son potencialmente modificables); contribuyendo a la creación de ambientes más saludables para la infancia (20-23).

Los ítems evaluados en la hoja verde del embarazo y lactancia son: factores socioeconómicos, antecedentes obstétricos-reproductivos, radiación ionizante, farmacia (parafarmacia, homeopatía y suplementos vitamínicos), exposiciones laborales, hobbies o aficiones de riesgo químico, sustancias psicoactivas legales e ilegales, hogar, exposiciones a pesticidas intra/extra-domiciliaria y percepción de riesgo medioambiental de los padres en el hogar y/o comunidad. La utilización de este formato en países como México y Argentina arrojan datos de relevancia en aspectos de consumo preconcepcional de alcohol de un $26.7 \%$ y $83.3 \%$, con mantenimiento de consumo de un $6.2 \%$ y $75.2 \%$ durante su embarazo, respectivamente $(24,25)$.

La adaptación e implementación de esta herramienta en diferentes contextos culturales, facilita el abordaje íntegro y preventivo de los factores medioambientales durante el embarazo. En este contexto, surge el proyecto Salud Ambiental para el Embarazo, Lactancia y Crianza en Iberoamérica (SAELCI), como una estrategia de alianza y de trabajo en red, para desarrollar este proyecto con el objetivo de identificar factores medioambientales, desde la etapa periconcepcional hasta la infancia, utilizando como herramienta compartida la hoja verde de salud ambiental; lo que posteriormente permitirá el desarrollo de proyectos que promuevan la salud infantil. Así, el objetivo de este estudio es identificar los factores medioambientales, desde la etapa periconcepcional hasta la infancia, utilizando como herramienta compartida la hoja verde de salud ambiental en Santander.

\section{Metodología}

Se realizó un estudio observacional, descriptivo de corte transversal. Se estimó una muestra a conveniencia de 204 mujeres con un poder del $80 \%$, por muestreo por captación, incluyendo gestantes y/o lactantes, adscritas a programas de atención a la gestante y/o lactante en cualquier edad gestacional o puerperio, de municipios del departamento de Santander-Colombia (Barrancabermeja, Málaga, Valle de San José, Zapatoca, Puente Nacional, Bucaramanga y Piedecuesta). Para participar en el estudio se requirió un consentimiento $\mathrm{y} / \mathrm{o}$ asentimiento informado.

La hoja verde fue validada por un equipo de pares expertos locales, incluyendo médico, enfermera, pediatra, trabajador social y epidemiólogo. Posteriormente, fue adaptada y piloteada en el contexto local con mujeres voluntarias de la ciudad de Bucaramanga y Medellín. La herramienta fue administrada en una entrevista clínica cara a cara por personal de enfermería previamente capacitado a través del curso iberoamericano de medio ambiente y salud reproductiva: taller sobre la hoja verde de embarazo y lactancia, el cual se desarrolló de manera virtual con el apoyo de la Pediatric Enviromental Health Special Unit (PEHSU) de la clínica virgen de la Arraixaca en Murcia. Luego de la aplicación de la herramienta, se hicieron las recomendaciones individuales de acuerdo a los hallazgos.

Se realizó un análisis estadístico descriptivo, en el cual se reportan frecuencia, proporciones, medias y desviaciones estándar, de acuerdo a la naturaleza de la variable. Se aplicó la prueba de Chi2 $\left(\chi^{2}\right)$ y Pearson para la comparación de los datos. Los datos fueron digitados en Excel y analizados en el paquete estadístico Stata 13.

Este estudio se desarrolló bajo la aprobación y supervisión del Comité Institucional de Ética en Investigación de la Universidad Autónoma de Bucaramanga. Todos los participantes firmaron el consentimiento informado antes de la entrevista.

\section{Resultados}

Se incluyeron 204 mujeres gestantes y/o lactantes con edades de embarazo entre 13 a 46 años y una edad promedio de 25.12 años $(\mathrm{DE} \pm 5.9)$. En cuanto al nivel de estudio, el $17.65 \%(\mathrm{n}=36)$ de las madres presentó la secundaria incompleta o menos, el 50\% (n=102) secundaria completa, y el $32.35 \%$ ( $n=66$ ) formación profesional. En los padres, se encontró que el $21 \%(\mathrm{n}=43)$ presentó una secundaria incompleta o menos, el 52\% ( $\mathrm{n}=106)$ secundaria completa y el $27 \%(n=55)$ formación profesional (Tabla 1).

Por otra parte, se observó un promedio de 36.7 ( $\mathrm{DE} \pm 5.6)$ semanas gestacionales (mín: 8 y máx: 42 semanas); donde el $53.9 \%$ de las madres $(\mathrm{n}=110)$ refirieron no tener intención de quedar en embarazo, el 6.4\% ( $\mathrm{n}=13)$ no lo había planteado y el 39.7\% (n=81) restante sí tuvo intención de estar en 
Tabla 1. Datos sociodemográficos.

\begin{tabular}{|c|c|c|}
\hline Nivel Académico & Madre n (\%) & Padre n (\%) \\
\hline Primaria Incompleta & $1(0.49)$ & $6(2.95)$ \\
\hline Primaria Completa & $24(11.76)$ & $28(13.73)$ \\
\hline Secundaria Incompleta & $11(5.39)$ & $9(4.41)$ \\
\hline Secundaria Completa & $102(50.00)$ & $106(51.96)$ \\
\hline Técnico & $22(10.78)$ & $20(9.8)$ \\
\hline Tecnólogo & $17(8.33)$ & $10(4.9)$ \\
\hline Universitario & $25(12.25)$ & $23(11.27)$ \\
\hline Especialista & $1(0.49)$ & $1(0.49)$ \\
\hline Maestría & $1(0.49)$ & $1(0.49)$ \\
\hline Total & $204(100.00)$ & $200(100.00)$ \\
\hline Consumo de cigarrillo preconcepcional & $10(4.90)$ & $39(19.1)$ \\
\hline Consumo de alcohol preconcepcional & $99(48.5)$ & $126(61.7)$ \\
\hline Procedencia & \multicolumn{2}{|c|}{ Sujetos n (\%) } \\
\hline Bucaramanga & \multicolumn{2}{|c|}{$40(19.6)$} \\
\hline Barrancabermeja & \multicolumn{2}{|c|}{$39(19.11)$} \\
\hline Málaga & \multicolumn{2}{|c|}{$40(19.6)$} \\
\hline Piedecuesta & \multicolumn{2}{|c|}{$11(5.4)$} \\
\hline Puente Nacional & \multicolumn{2}{|c|}{$26(12.75)$} \\
\hline Valle de San José & \multicolumn{2}{|c|}{$17(8.33)$} \\
\hline Zapatoca & \multicolumn{2}{|c|}{$31(15.2)$} \\
\hline
\end{tabular}

embarazo. Además, el 6.4\% (n=13) utilizó tratamiento para lograr el embarazo, como fecundación in vitro o estimulación ovárica.

En cuanto a los tipos de vivienda, el $65.2 \%(\mathrm{n}=133)$ de los cuidadores vivían en casa, y un $29.4 \%(n=60)$ en apartamento. Entre los materiales utilizados para la construcción de las viviendas, se observó baldosa con un $80.8 \%(n=165)$, cemento con el $17.7 \%(n=36)$ y tierra o madera con el $1.5 \%(n=3)$. Para la construcción del techo el material más utilizado fue placa con un $52.4 \%(\mathrm{n}=107)$; y el material menos utilizado el plástico con un $2.5 \%(\mathrm{n}=5)$ (Tabla 2).

Por otra parte, durante la etapa preconcepcional el 48.5\% $(\mathrm{n}=99)$ de las gestantes reportaron consumo de alcohol, y entre estas, el 91.9\% $(n=91)$ consumía cerveza, el 30.3\% $(\mathrm{n}=30)$ bebidas con 40 grados de alcohol como ron o aguardiente; el $9.09 \%(n=9)$ vino y cerveza sin alcohol, y el $2.02 \%(n=2)$ bebidas con hasta 25 grados de alcohol como cremas. Durante el embarazo, el $45.73 \%$ de las madres reportaron una disminución de consumo de alcohol. Con respecto a la frecuencia de consumo de cerveza el $31.37 \%$ $(\mathrm{n}=64)$ de las mujeres consumían 1 vez al mes un promedio de 4.96 cervezas por mes; el $11.76 \%$ de las mujeres consumían una vez por semana un promedio de 6.37 cervezas por vez ( $\mathrm{DE}= \pm 10.02$, mín: 1 y máx: 50 ) (Tabla 3).

En los padres se encontró que el 61.76\% (n=126) refirieron un consumo de alcohol durante la etapa de la espermatogénesis, prefiriendo el consumo de cerveza (59.31\%), seguido por las bebidas con 40 grados de alcohol como ron o aguardiente (25\%), y finalmente las bebidas con hasta 25 grados de alcohol como cremas (4.41\%), vino $(3.92 \%)$ y cerveza sin alcohol $(1.96 \%)$. Con respecto a la frecuencia de consumo de cerveza en los padres se evidenció que el $40,19 \%$ ( $n=82)$ la consumían una vez al mes, y en este grupo se observó un promedio 8.24 cervezas por vez $(\mathrm{DE}=$ 5.38, mín: 2 y máx: 30 ), seguido por quienes consumen una vez por semana con un promedio de 7.37 cervezas por vez (DE=6.40, mín: 1 y máx: 30) (Tabla 4).

Durante el embarazo, se observó una diferencia estadísticamente significativa del consumo mensual de alcohol entre padres (66.18\%) y madres (27.94\%), una o más veces al mes $(\mathrm{p}=0.000)$. 
Tabla 2. Descripción de los tipos de elementos de construcción de las viviendas.

\begin{tabular}{|c|c|c|}
\hline Tipo vivienda & $n$ & $\%$ \\
\hline Apartamento & 60 & 29.4 \\
\hline Casa & 133 & 65.2 \\
\hline Finca & 10 & 4.9 \\
\hline Asentamiento & 1 & 0.5 \\
\hline Total & 204 & 100 \\
\hline \multicolumn{3}{|l|}{ Material Piso } \\
\hline Baldosa & 165 & 80.8 \\
\hline Cemento & 36 & 17.7 \\
\hline Tierra & 2 & 1 \\
\hline Madera & 1 & 0.5 \\
\hline Total & 204 & 100 \\
\hline \multicolumn{3}{|c|}{ Material Paredes } \\
\hline Frisado & 168 & 82.4 \\
\hline Ladrillo & 21 & 10.3 \\
\hline Adobe & 7 & 3.4 \\
\hline Otro & 8 & 3.9 \\
\hline Total & 204 & 100 \\
\hline \multicolumn{3}{|l|}{ Material Techo } \\
\hline Placa & 107 & 52.4 \\
\hline Plástico & 5 & 2.5 \\
\hline Eternit & 56 & 27.4 \\
\hline Madera & 21 & 10.3 \\
\hline Otro & 15 & 7.4 \\
\hline Total & 204 & 100 \\
\hline
\end{tabular}

Tabla 3. Consumo promedio de alcohol de la madre, antes del embarazo.

\begin{tabular}{|c|c|c|c|}
\hline \multirow[t]{2}{*}{ Bebida alcohólica } & \multirow[t]{2}{*}{$\mathrm{n}$} & \multicolumn{2}{|c|}{ Frecuencia de consumo (No. de veces) } \\
\hline & & Semanal & Mensual \\
\hline \multirow[t]{2}{*}{ Cerveza* } & 91 & $\begin{array}{c}6.37 \\
(D E=10.02, \min =1, \text { máx }=50) \quad(n=24)\end{array}$ & $\begin{array}{c}4.96 \\
\left(\mathrm{DE}=4.58, \min ^{\prime}=1, \text { máx }=30\right) \\
(n=64)\end{array}$ \\
\hline & & $\begin{array}{l}2.66 \text { cervezas de } 2 \text { a } 4 \text { veces } \\
(D E=1.15, \text { min=2, máx=4) }(n=3)\end{array}$ & \\
\hline Vino & 9 & $\begin{array}{c}3 \\
(\min =1, \text { máx }=8)(n=8)\end{array}$ & $\begin{array}{c}6 \\
(\min =6, \text { máx }=6) \\
(n=1)\end{array}$ \\
\hline Aguardiente & 30 & $\begin{array}{c}4.30 \\
(D E=3.06, \min =1, \text { máx }=10) \quad(n=13)\end{array}$ & $\begin{array}{c}6.88 \\
(\mathrm{DE}=8.60, \text { min=1, máx=35) }(n=17)\end{array}$ \\
\hline Cerveza sin alcohol & 9 & 0 & $\begin{array}{c}2.33 \\
(D E=1.41, \min =1, \text { máx }=5)(n=9)\end{array}$ \\
\hline $\begin{array}{l}\text { Bebidas con hasta } \\
25 \text { grados de alcohol } \\
\text { como cremas }\end{array}$ & 2 & $\begin{array}{c}3 \\
(\min =3, \text { máx=3) }(n=1)\end{array}$ & $\begin{array}{c}4 \\
(\min =4, \text { máx=4) }(n=1)\end{array}$ \\
\hline
\end{tabular}


Tabla 4. Consumo promedio de cerveza del padre, antes del embarazo.

\begin{tabular}{lccc}
\hline Frecuencia & $\begin{array}{c}\text { Cervezas } \\
\text { por vez }\end{array}$ & DE & $\begin{array}{c}\text { Mínimal } \\
\text { Máxima }\end{array}$ \\
\hline 1 vez al mes $(n=82)$ & 8.24 & \pm 5.38 & $2 / 30$ \\
\hline 1 vez a la semana $(n=27)$ & 7.37 & \pm 6.40 & $1 / 30$ \\
\hline 2 a 4 veces por semana $(n=4)$ & 20 & \pm 13.54 & $10 / 40$ \\
\hline 5 a 6 veces por semana $(n=2)$ & 3.5 & \pm 0.7 & $3 / 4$ \\
\hline Diario $(n=3)$ & 11.66 & \pm 7.63 & $3 / 24$ \\
\hline 2 a 3 veces por día $(n=1)$ & 15 & $n / a$ & $15 / 15$ \\
\hline 4 a 5 veces por día $(n=1)$ & 1 & $n / a$ & $1 / 1$ \\
\hline Más de 6 veces por día $(n=1)$ & 3 & $n / a$ & $3 / 3$ \\
\hline${ }^{*}$ na: no aplica. & & &
\end{tabular}

En cuanto al consumo de cigarro por parte de la madre, se encontró que el $4.90 \%$ de las madres fumaban durante etapas periconcepcionales un promedio 2.03 cigarros por día ( $\mathrm{DE}=4.35$, mín: 1 y máx: 20). El $28.95 \%$ de las madres reportaron una disminución en el consumo de tabaco durante el embarazo, y de estas un $23.5 \%$, reportó abandono del consumo del tabaco.

Respecto a los padres, el $19.1 \%$ fumaba durante la etapa periconcepcional con un promedio de 4.55 cigarrillos por día ( $\mathrm{DE}=6.7$, mín: 1 y máx: 30 ). El promedio de edad de inicio fue de 11.6 años. El $42.9 \%$ de los padres cambió el consumo debido al embarazo. La exposición pasiva a tabaco de la madre, fue percibida como alta en el $17.2 \%$ de los casos. Durante el embarazo no se observó una diferencia estadísticamente significativa del cambio de consumo de tabaco entre padres $(9.71 \%)$ y madres $(5.34 \%)(\mathrm{p}=0.329)$.

\section{Discusión}

El aumento de la preocupación de la sociedad científica por el impacto de los factores ambientales en el desarrollo de los niños, hace necesario el desarrollo de evidencia científica que permita determinar los factores de riesgo latentes y las potenciales alternativas de cuidado; sin embargo, las características culturales de la sociedad actual y su diferencia entre poblaciones rurales y urbanas, pueden limitar la recolección de la información requerida de padres y cuidadores de los menores, o potenciar la presencia de sesgos como de admisión, memoria o no respuesta, los cuales fueron tenidos en cuenta en el proyecto y buscaron modularse a través de su estructura metodológica y análisis de resultados.

Los factores medioambientales evaluados y características sociodemográficas fueron similares a otros estudios en
Latinoamérica, por ejemplo, la edad de la madre fue similar a lo observado por autores como Bermúdez (24) Moraes (25), donde la población tuvo una media de 24.67 años (mín: 13 y máx: 42 años). Con respecto al nivel académico, este tiende a ser similar a los datos arrojados por Ortega-García 2015 (28), con algunas diferencias porcentuales de relevancia con Bermúdez 2015, (24) pues aunque la población analizada en este proyecto era de procedencias similares, la proporción de formación académica si presenta diferencias que pueden influir en otros de los resultados.

Respecto a la planificación del embarazo, al contrastar con los resultados reportados por Moraes 2016 (25) donde el embarazo no fue planificado por el $57 \%$ de las mujeres $(n=182)$, se observa que al juntar las mujeres que no lo habían planteado, con quienes no habían tenido intención de estar en embarazo un $60.29 \%(n=123)$ hay similitud en este aspecto comportamental de la población, que puede contribuir con los factores de riesgo para madre e hijo.

Por otra parte, en cuanto a los tipos de vivienda, los diferentes autores revisados no enuncian estas descripciones de cuidadores de niños de menos de cinco años. Se observó que los materiales de la vivienda como pisos de madera y tierra, paredes de adobe $u$ otros, y techos de madera u otros, representan más del $10 \%$ de los elementos en los cuales fueron construidas las viviendas de los cuidadores de este estudio; materiales que de acuerdo a otros autores, incrementan el riesgo para la presencia y reproducción de plagas y vectores (29).

En cuanto a los datos de consumo de alcohol periconcepcional, los resultados difieren en múltiples estudios, de acuerdo al país donde se han realizado; en México, según Bermúdez et al., el consumo en las mujeres es del $26.7 \%$ (24); y en Uruguay según Moraes es de 23.82\% (25), valores muy similares, pero inferiores en contraste a los encontrados 
en este trabajo, que refieren un consumo mayor en la población objeto, lo cual podría aumentar el riesgo en la salud ambiental de los menores. Sin embargo, en Argentina según López et al., se observó un consumo en el 75.2\% de las mujeres (26), evidenciando mayor consumo de lo observado en la población objeto de este estudio.

Aunque la hoja verde permite identificar el consumo de alcohol, de acuerdo a lo encontrado por Manich et al., el que el $16.12 \%$ de las mujeres negaron consumo de alcohol en el embarazo, se encontraron biomarcadores de esta sustancia en meconio en este grupo (27), lo que refiere que el uso único de cuestionarios como herramienta de cribado para descartar la exposición de alcohol en el embarazo, no se debe considerar como herramienta eficiente, por lo que se recomienda la realización de proyectos que determinen con marcadores moleculares la eficiencia del cuestionario.

Al revisar el tipo de licor consumido, se evidencia que al igual que la población mexicana, el más consumido es la cerveza con un $65.2 \%$, sin embargo, en la población objeto de este estudio fue mucho más alta la proporción. Con respecto al consumo de otros tipos de bebidas, en el estudio realizado en México por Bermúdez et al., ocupó el segundo lugar el consumo de combinaciones de cerveza con 17.5\% (el cual no fue analizado en la adaptación de la hoja verde de nuestra región), seguidos de Brandy con $13 \%$ y vino con el $4.3 \%$, que en comparación con este estudio se observó un comportamiento similar en el consumo de estas bebidas, con mayores proporciones (24). Este factor podría afectar el estado de salud medioambiental de la población menor de 5 años.

En el análisis de la reducción del consumo, luego de conocer su estado de embarazo, encontramos que aunque en países como México con un $93.8 \%$ (24) y Argentina con $83.7 \%$ (26), es evidente que la mayoría de los cambios se atribuyen al embarazo, puesto que se produjeron al momento que las mujeres se enteraron de que se encontraban en gestación; este comportamiento fue similar en este estudio, sin embargo el 74.05\% mostraron una menor intención por la reducción de consumo, lo cual podría aumentar el riesgo en la salud de la diada madre e hijo.

Otro factor importante es el consumo de tabaco, muchos autores reafirman la utilidad de asesorías para la cesación del mismo, dado que su consumo periconcepcional, está asociado con múltiples efectos adversos al nacer, como nacimiento pretérmino, muerte fetal in vitro o bajo peso al nacer (30); o complicaciones en el desarrollo como bajo coeficiente intelectual que puede generar trastornos en el aprendizaje, el habla o un déficit de atención (31). Respecto a la edad promedio de inicio para fumar de la madre fue de 17 años (DE=2.79, mín: 14 y máx: 22) la cual está por encima del promedio mundial (13 a 15 años) (32), pero dentro del promedio nacional (12 a 17 años) (33). Los resultados del mayor consumo promedio de tabaco, al igual que otros autores (34), muestran que el $34.3 \%$ de las mujeres gestantes, consume entre 1 a 5 cigarros día.
Finalmente, respecto a la exposición pasiva al tabaco, se evidenció que esta es mucho más baja en comparación con otras poblaciones, de acuerdo a lo mostrado por Ortega la población que refiere nada o poco de exposición pasiva es del $72.6 \%$ (28), mientras que esa misma población en este estudio, corresponde al $82.84 \%$ de la población objeto.

\section{Conclusiones}

Este estudio aporta evidencia científica sobre el tema en el ámbito local, y traslada una herramienta válida de abordaje de salud medioambiental y los riesgos al contexto Colombiano. Se recomienda la aplicación de este instrumento en el abordaje integral de la consulta de atención madre-hijo, tal como se lleva a cabo en otros contextos internacionales, para favorecer la oportuna detección de dichos factores.

Los datos sugieren la presencia de consumo de alcohol periconcepcional y que, a su vez, el estado de embarazo favorece la disminución de consumo; por lo que el uso de herramientas como la hoja verde, suministran información para el planteamiento de programas educativos que permitan el reforzamiento de los cursos de preparación para la maternidad y paternidad, y la cesación del consumo de tabaco y alcohol en esta población; programas dirigidos a prevenir la enfermedad y promover la salud infantil asociada a factores ambientales de nuestra población.

En futuros estudios se recomienda determinar la correlación entre las variables estudiadas, como los materiales con los que están construidas las viviendas, con los antecedentes clínicos de los cuidadores, para establecer las correlaciones existentes con la salud ambiental infantil.

\section{Conflicto de intereses}

Los autores declaran no tener conflicto de interés.

\section{Agradecimientos}

Este trabajo fue llevado a cabo gracias al apoyo de la convocatoria interna (E56002) de la Universidad Autónoma de Bucaramanga, y al apoyo logístico y administrativo de los estudiantes: Fabián Plata, Natalia Bonilla, Claudia Mejía y Carolina Rojas.

\section{Referencias}

1. Carlson J, Tamburlini G. Policy development. En: Tamburlini G, Von Ehrenstein OS, Bertollini R, editors. Children's Health and Environment: A review of evidence. Copenhagen: WHO Regional Office for Europe. 2002: 207-18.

2. Dunn AM, Burns C, Sattler B. Environmental health of children.J Pediatr Health Care. 2003; 17: 223-31. 
3. Ortega JA, Cárceles-Álvarez A, Vicente-Calderón C. Trastorno por déficit de naturaleza: ¿hipótesis o realidad?. Urgencias en pediatría. 2015; 12 (1): 2-3.

4. Guidotti TL; Gitterman BA. Global pediatric environmental health. Pediatr Clin North Am. 2007; 54(2): 335-50.

5. Valenzuela, Patricia M; Matus, M. Soledad; Araya, Gabriela I; Paris, Enrique. Pediatría ambiental: Un tema emergente / Environmental pediatrics: an emerging issue. J Pediatr (Rio J). 2011; 87(2): 89-99.

6. Walker B. Pediatric environmental health. J Natl Med Assoc. 2005; 97(2): 262-9.

7. Paris E, Bettini M, Molina H, Mieres J, Bravo V, et al. La importancia de la salud ambiental y el alcance de las unidades de pediatría ambiental. Rev Med Chile. 2009; 137: 101-105

8. Landrigan PJ. Children as a vulnerable population. Int J Occup Med Environ Health. 2004; 17(1): 175-7.

9. Ortega García JA, Ferrís i Tortajada J, Claudio Morales $\mathrm{L}$, Berbel Tornero O. Pediatric environmental health specialty units in Europe: from theory to practice. An Pediatr 2005; 63:143-51.

10. Herrmann M, King K, Weitzman M. Prenatal tobacco smoke and postnatal secondhand smoke exposure and child neurodevelopment. Curr Opin Pediatr. 2008; 20(2):184-90.

11. Lee BE, Hong YC, Park H, Ha M, Kim JH, Chang N, Et al. Secondhand smoke exposure during pregnancy and infantile neurodevelopment. Environ Res. 2011; 111(4):539-44. Disponible en: https://doi.org/10.1016/ j.envres.2011.02.014

12. McCollough $\mathrm{CH}$, Schueler BA, Atwell TD, Braun NN, Regner DM, Brown DL, LeRoy AJ. Radiation exposure and pregnancy: when should we be concerned? Radiographics. 2007; 27:909-17

13. Ortega García JA, Angulo MG, Sobrino-Najul EJ, Soldin OP, Mira AP, Martínez-Salcedo E, Claudio L. Prenatal exposure of a girl with autism spectrum disorder to 'horsetail' (Equisetum arvense) herbal remedy and alcohol: a case report. J Med Case Rep. 2011; 5: 129.

14. McDiarmid MA, Gardiner PM, Jack BW. The clinical content of preconception care: environmental exposures. Am J Obstet Gynecol. 2008; 199(6 Suppl 2):S357-61.

15. Ortega-García JA, Gutierrez-Churango JE, SánchezSauco MF, Martínez-Aroca M, Delgado-Marín JL, Sánchez-Solis M, et al. Head circumference at birth and exposure to tobacco, alcohol and illegal drugs during early pregnancy. Childs Nerv Syst. 2012; 28: 433-9.

16. World Health Organization. The paediatric environmental history Recording children's exposure to environmental health threats: A "green page" in the medical record

17. Ortega García JA, et al. La historia clínica medioambiental y la "hoja verde" en la consulta de Pediatría de Atención Primaria. Pediatría Integral. 2007; 20-23

18. Gobierno de la ciudad de Buenos Aires. Hoja Pediátrica de pesquisa del riesgo ambiental

19. The National Environmental Education Foundation, Pediatric Environmental History. The Screening Environmental History.

20. Ortega García JA, Sánchez Sauco MF, Jaimes Vega DC, Pernas Barahona A. Hoja verde. Creando ambientes más saludables durante el embarazo y lactancia materna. Unidad de Salud Medioambiental Pediátrica. Murcia, España; 2013.

21. Ortega García JA, Sánchez Sauco MF, Jaimes Vega DC, Pernas Barahona A. Manual de la Hoja Verde de
Salud Medioambiental Reproductiva. Creando ambientes más saludables durante el embarazo y lactancia. Unidad de Salud Medioambiental Pediátrica. Versión 1.0. Murcia, España; 2013.

22. Ortega García JA, Ferris i Tortajada J, Claudio Morales L, Berbel Tornero O. Unidades de Salud Medioambienta Pediátricas en Europa: de la teoría a la acción. An Pediatr (Barc). 2005; 63: 143-51.

23. Ortega Garcia JA, Sanchez Sauco MF, Jaimes Vega DC, Pernas Barahona A. Hoja verde. Creando ambientes más saludables durante el embarazo y lactancia materna. Unidad de Salud Medioambiental Pediátrica. Murcia, España; 2013. Disponible en: http://pehsu.org/wp/ ?page id=349

24. Bermúdez RS, Linares $B$, Serrano MG, Álvarez V. Proyecto saelci-guanajuato. Exposición al alcohol periconcepcional y salud del recién nacido en el hospital materno infantil de Irapuato. Verano de la Investigación Científica. 2016; 2(1): 1536-1540.

25. Moraes M, Duarte MV, Barceló JG, Báez P, González G, Sosa C. Consumo de tabaco, alcohol y marihuana según auto declaración en mujeres que tuvieron su parto en el Centro Hospitalario Pereira Rosell (mayo 2013-abril 2014). Revista Médica de Uruguay. 2016; 32(4): 234-241.

26. López MB, Arán Filippetti V, Cremonte M. Consumo de alcohol antes y durante la gestación en Argentina: prevalencia y factores de riesgo. Rev Panam Salud Pública. 2015; 37(4/5):211-7.

27. Manicha A, Velascoa M, Joyaa X, García-Larab N.R, Pichinic S, Vall O, Et all. Validez del cuestionario de consumo materno de alcohol para detectar la exposición prenatal. Anales de Pediatría (Barcelona). 2012; 76(6):324-328.

28. Ortega-García JA, Cárceles-Álvarez A, CottonCaballero A, Pastor-Torres E, Cánovas-Conesa C.A, Martínez-Cayuelas E, et al. Factores ambientales relacionados con la duración de la lactancia: estudio de seguimiento a 1 año. Acta Pediátrica Española. 2015; 73(4): 97-104.

29. Organización Panamericana de la Salud. Guía: Hacia una Vivienda Saludable - Guía para el facilitador; Los Pinos 259, Urb. Camacho, Lima 12 - Perú. Disponible en: http://www.bvsde.paho.org/texcom/cd045364/ vivsal.pdf

30. Ministerio de Salud y Protección Social - Colciencias. Guía de Práctica Clínica para la prevención, detección temprana y tratamiento del embarazo, parto o puerperio, Guías No. 11-15.2013

31. Ortega J.A., Ferrís J, Berbel O, Romero K.J, Rubalcava L, Martínez E, et all. Neurotóxicos medioambientales $(\mathrm{IV})^{*}$. Tabaco, alcohol, solventes, flúor y aditivos alimentarios: efectos adversos en el sistema nervioso fetal y posnatal y medidas preventivas. Acta Pediatr Esp. 2006; 64(10): 493-502.

32. The Global Youth Tobacco Survey Collaborative Group. Tobacco use among youth: a cross country comparison. Tob. Control. 2002; 11(3): 252-270.

33. Ministerio de la Protección Social. Encuesta Nacional de Salud 2007. Resultados Nacionales. Resultados. Bogotá: Ministerio de la Protección Social. Cendex; 2009. Report No.: ISBN 978-958-716-187-8.

34. Aranda JM, Mateos P, González A, Sánchez F, Luna del Castillo JD. Validez de distintas medidas de consumo de tabaco durante el embarazo: especificidad, sensibilidad y puntos de corte dónde y cuándo. Revista Española de Salud Pública. 2008; 82(5). 